\title{
Structural, Optical, and Magnetic Properties of Co Doped CdTe Alloy Powders Prepared by Solid-State Reaction Method
}

\author{
M. Rigana Begam, N. Madhusudhana Rao, Girish M. Joshi, S. Kaleemulla, \\ M. Shobana, N. Sai Krishna, and M. Kuppan \\ Thin Films Laboratory, Materials Physics Division, School of Advanced Sciences, VIT University, Vellore 632 014, India \\ Correspondence should be addressed to N. Madhusudhana Rao; drnmrao@gmail.com
}

Received 30 May 2013; Accepted 14 August 2013

Academic Editor: Kim Chow

Copyright (c) 2013 M. Rigana Begam et al. This is an open access article distributed under the Creative Commons Attribution License, which permits unrestricted use, distribution, and reproduction in any medium, provided the original work is properly cited.

Co doped CdTe powder samples were prepared by solid-state reaction method. In the present work effect of Co doping on structural, optical, and magnetic properties has been studied. X-ray diffraction studies confirm zinc blend structure for all the samples. The lattice parameter showed linear increase with the increase in Co content. The elemental constituents were characterized by EDAX. Optical studies showed the increase in band gap with increase in Co level. The samples were diluted magnetic semiconductors and exhibited clear hysteresis loop showing room temperature ferromagnetism as confirmed by vibrating sample magnetometer.

\section{Introduction}

Doping of magnetic atoms like $\mathrm{Mn}, \mathrm{Fe}, \mathrm{Cr}, \mathrm{Co}, \mathrm{Ni}$, and so forth into II-VI, II-V, III-V, and IV-VI host semiconductors leads to a new class of materials called dilute magnetic semiconductors (DMS) [1]. In recent years, considerable amount of research work has been devoted to form new DMS and to improve the ferromagnetic properties of the known DMS in an effort to develop new spintronic devices, such as spin valves, spin light emitting diodes, magnetic sensors, logic devices, and ultrafast optical switches [2]. The unique properties of DMS attracted the scientific community and are extensively studied for their distinctive behavior in spintronic devices which allow control of both the spin and the charge of carriers [3,4]. II-VI DMS systems are particularly interesting, since doping of magnetic ions in II-VI systems is more effective than metal oxide systems. Ferromagnetic semiconductors formed in this manner by varying the concentration of the magnetic material received much attention as the presence of magnetic ions allows tailoring the energy gap and lattice constant leading to a number of unusual electronic and magnetic properties resulting from the large $\mathrm{sp}-\mathrm{d}$ exchange interaction between the magnetic ions and the band electrons. These interactions are strongly influenced by the ground state of the particular substituted magnetic ion $[5,6]$. In order to realize the practical implication of spin-based devices, DMS must exhibit room temperature ferromagnetism. Though room temperature ferromagnetism was reported in II-VI DMS in both experimentally and theoretically investigations by several researchers, the exact origin of magnetism in DMS is still a challenge to scientific community. II-VI based DMS with $\mathrm{Mn}$ as the dopant has been studied extensively, but the studies with other magnetic dopants like $\mathrm{Fe}, \mathrm{Co}, \mathrm{Ni}$, and so forth are meager. Limited number of reports are available on Co doped CdTe crystals. This could be due to the difficulties in preparing homogeneous crystals with sufficient amounts of substituted $\mathrm{Co}$ as its solubility is very low. Magnetic properties of Co based CdTe materials have been studied [79] and the large $\mathrm{p}-\mathrm{d}$ interaction and $\mathrm{d}$-d exchange interaction were established between $\mathrm{Co}^{2+}$ and $\mathrm{Cd}^{2+}$ ions [10-12]. Studies on Co doped CdTe alloy powders have not been reported so far. These factors and the interesting properties exhibited by Co doped CdTe system are the motivation to carry out this study. In the present work, the structural, optical, and magnetic properties on Co doped CdTe alloy powders prepared by solid-state reaction method have been reported. 


\section{Experimental}

Co doped CdTe powders with Co concentrations of 5 at.\% and 8 at.\% were synthesized by single step solid-state reaction method. $5 \mathrm{~N}$ purity CdTe, Co, and Te (99.99\%, M/S SigmaAldrich) were used as source materials. Appropriate quantities of freshly prepared CoTe and CdTe were mixed and ground thoroughly for 16-18 hours to ensure homogeneity and then sintered at $500^{\circ} \mathrm{C}$ for 6 hours under a pressure of $10^{-3}$ Torr. The sintered Co doped CdTe alloy powder were characterized by different techniques. Powder X-ray diffraction (XRD) patterns of all the samples were recorded using X-ray diffractometer (Bruker, D8 Advance), equipped with $\mathrm{Ni}$ filter, and operated at $40 \mathrm{kV}, 30 \mathrm{~mA}\left(\mathrm{CuK}_{\alpha}\right.$ radiation, $\lambda=0.15406 \mathrm{~nm}$ ) with a scan speed of $0.02^{\circ} \mathrm{s}^{-1}$ in the $2 \theta$ range of $20^{\circ}-80^{\circ}$. The elemental composition of the samples was arrived at using EDAX (Genesis X4M attached to the JSM $840 \mathrm{~A})$. The room temperature diffuse reflectance spectra of the samples were recorded using double beam UV-Vis-NIR spectrophotometer (Jasco V-670) in the wavelength range of 200-2500 nm. Magnetic measurements were carried out using a vibrating sample magnetometer (Lakeshore VSM $7410)$ at an applied field of 15,000 Gauss at room temperature.

\section{Results and Discussion}

3.1. Structural Analysis. Powder XRD patterns of pure and Co doped CdTe alloy powders are shown in Figure 1. All the peaks seen in the pattern coincided with standard cubic CdTe structure (JCPDS Card no. 89-3053) and the subsequent patterns are indexed with their respective planes. No peak related to Co or CoTe was observed in the X-ray diffraction pattern after thorough analysis by Rietveld software confirming that Co has been incorporated into the crystal lattice of CdTe without changing the cubic structure of CdTe. It can be seen that all the samples exhibited zinc blend structure with (111) predominant orientation. Figure 2 shows variation of " $a$ " with Co. The lattice parameter was found to decrease linearly with increase in " $x$ " obeying Vegard's law. The decrease in lattice parameter with the increase in Co concentration implies the lattice contraction. Change in lattice parameter may be due to the difference in ionic radii of cobalt and cadmium. The ionic radii of $\mathrm{Co}^{2+}$ and $\mathrm{Cd}^{2+}$ in tetrahedral site are $(0.58 \AA)$ and $(0.78 \AA)$ [13] respectively. The difference between the two ionic radii is small. As a consequence, changes in lattice cell parameters and cell volume with cobalt substitution are also small. As the $\mathrm{Co}^{2+}$ ions were in a tetrahedral environment, these results a decrease in lattice parameter by considering the smaller ionic radius of $\mathrm{Co}^{2+}$ ions. Hence it can be concluded that the doped $\mathrm{Co}^{2+}$ ions substitute the host lattice at tetrahedral site symmetry that brings expansion in $\mathrm{CdTe}$ lattice.

3.1.1. EDAX Analysis. The spectra shown in Figure 3 depict the chemical composition of the Co doped CdTe alloy powders at 5 at.\% and 8 at.\%. The EDAX spectra revealed that the peaks corresponding to $\mathrm{Cd}, \mathrm{Te}$, and $\mathrm{Co}$ and their weight (wt.\%) ratio and atomic (at.\%) ratio compositions are
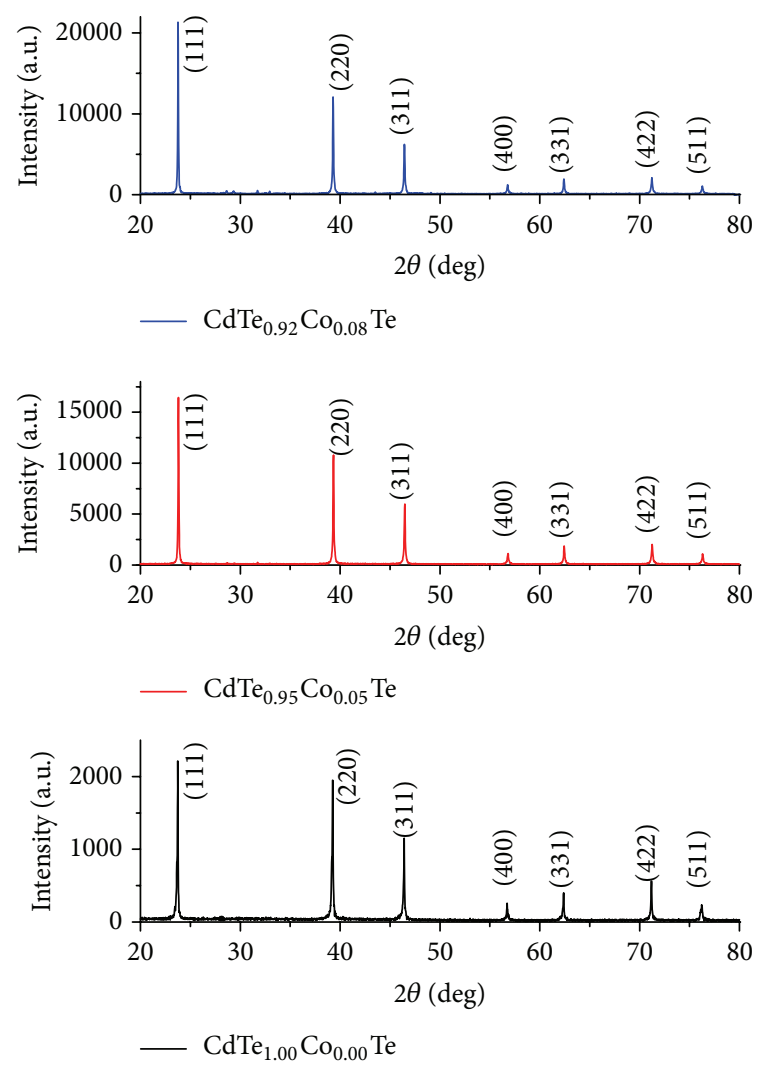

FIGURE 1: X-ray diffraction pattern of $\mathrm{Cd}_{1-x} \mathrm{Co}_{x}$ Te alloy powders.

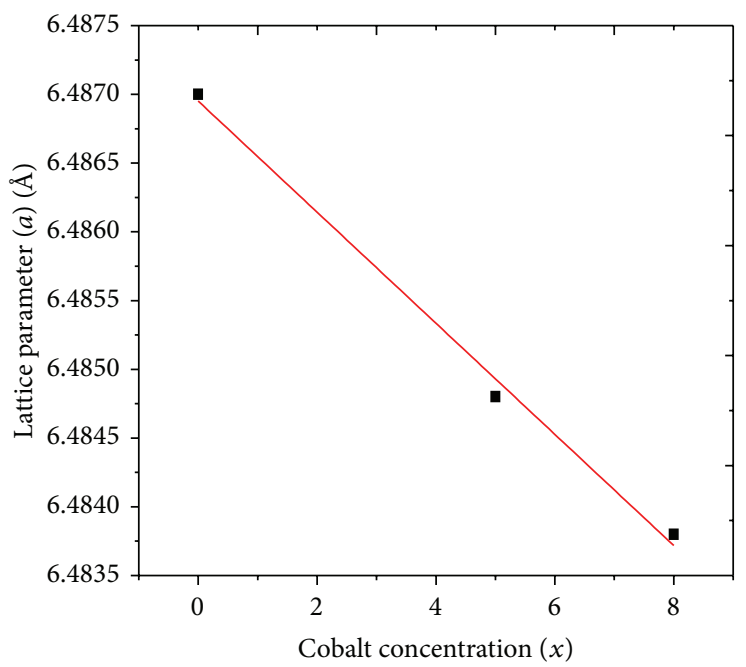

FIGURE 2: Variation of lattice parameter with increase in Co level.

shown in the inset of Figure 3. It is clear that the at.\% of Cd element decreases with increasing Co content indicating the formation of Co doped CdTe alloy powder as confirmed by powder XRD.

3.2. Optical Properties. Figure 4 shows the optical reflectance spectra of pure and Co doped CdTe alloy powders in the photon energy range of $1.0-2.5 \mathrm{eV}$. Inset of Figure 4 shows the expanded view of fundamental band edge $E_{0}$ with 


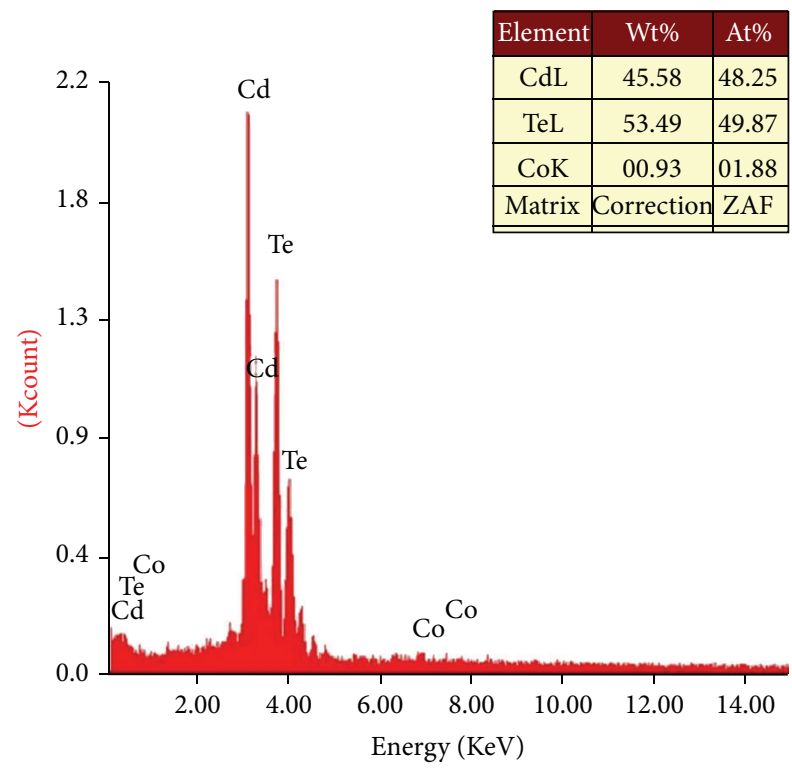

(a)

\section{Element $\quad \mathrm{Wt} \% \quad \mathrm{At} \%$}

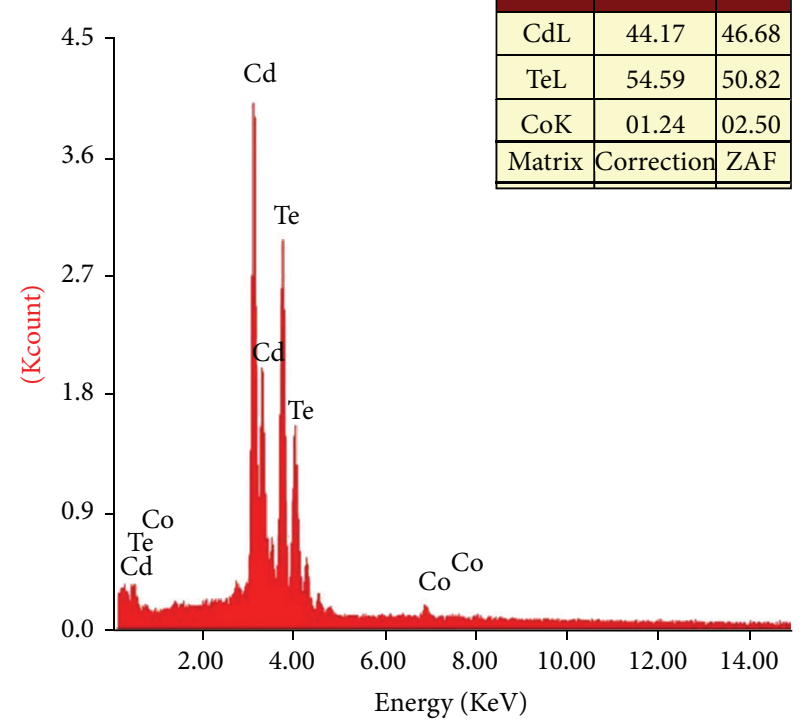

(b)

FIgURE 3: EDAX spectra of (a) $\mathrm{Cd}_{0.95} \mathrm{Co}_{0.05} \mathrm{Te}$ and (b) $\mathrm{Cd}_{0.92} \mathrm{Co}_{0.08} \mathrm{Te}$ alloy powders.

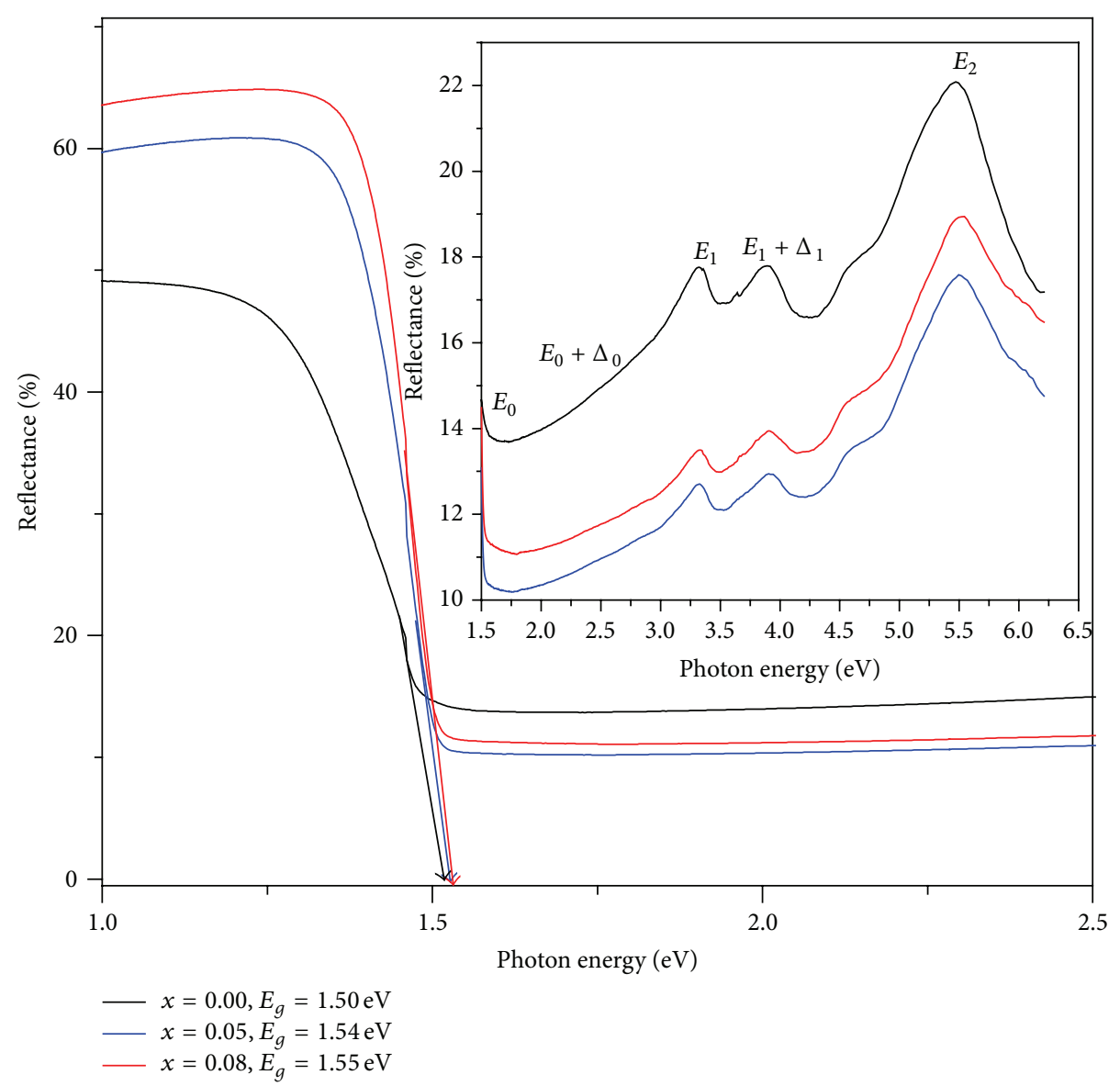

Figure 4: Plot of reflectance versus photon energy (eV) for pure and Co doped CdTe alloy powders. 


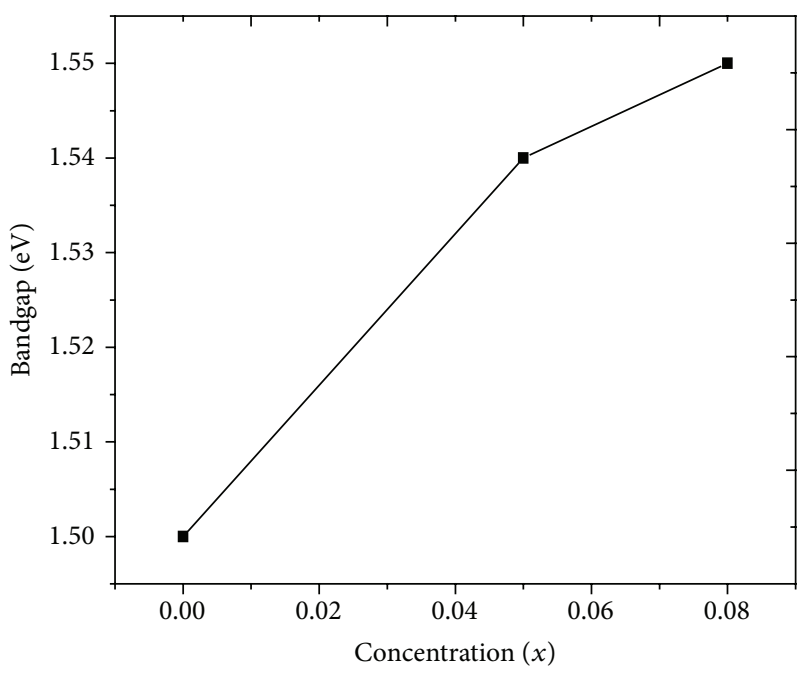

Figure 5: Variation of band gap of $\mathrm{Cd}_{1-x} \mathrm{Co}_{x}$ Te powders with Co concentration.

the observed electronic transitions labeled as $E_{0}, E_{0}+\Delta_{0}$, $E_{1}, E_{1}+\Delta_{1}$, and $E_{2}$ peaks. It is evident from Figure 4 that the fundamental absorption edge $\left(E_{0}\right)$ shifted systematically near the IR region towards higher energy with increase in Co level. This observed increase in absorption edges with increase in Co concentration indicates that the $\mathrm{Co}^{2+}$ ions have replaced the $\mathrm{Cd}^{2+}$ ions in the CdTe lattice. Variation of optical gap energy as a function of Co concentration is illustrated in Figure 5 and the optical band gap energy was found to increase with the increase in Co concentration. Kim et al. [14] have also reported similar variation optical band gap in $\mathrm{Zn}_{1-x} \mathrm{Co}_{x} \mathrm{O}$ thin films. In general, it is interpreted that the increase in the band gap in Co doped CdTe alloy powders may be due to sp-d exchange interactions between band electrons and localized d electrons of Co ions.

3.3. Magnetic Studies. The magnetic properties of pure and Co doped CdTe for 5 at.\% and 8 at.\% alloy powders were investigated by magnetization measurements carried out at room temperature. The $M-H$ curves of pure and Co doped $\mathrm{CdTe}$ are shown in Figure 6. Ferromagnetism in pure CdTe was not expected as it is diamagnetic in nature, whereas all the Co doped samples exhibited a well defined magnetization hysteresis confirming ferromagnetic behaviour at room temperature. It was observed from Figure 6 that the saturation magnetization increased with increase in Co concentrations. The obtained magnetization $\left(M_{s}\right)$ for 5 at.\% and 8 at.\% of Co are $0.00086 \mathrm{emu} / \mathrm{g}$ and $0.0054 \mathrm{emu} / \mathrm{g}$. Generally ferromagnetism in DMS is considered to originate from the exchange interaction between free delocalized carriers and the localized $\mathrm{d}$ spins on the Co ions [15]. Presence of free carriers is therefore necessary for the appearance of ferromagnetism. Alver et al. [16] have reported ferromagnetism in Co doped CdTe thin films at room temperature and at low temperatures, but the doping composition dependent magnetization study was not carried out.

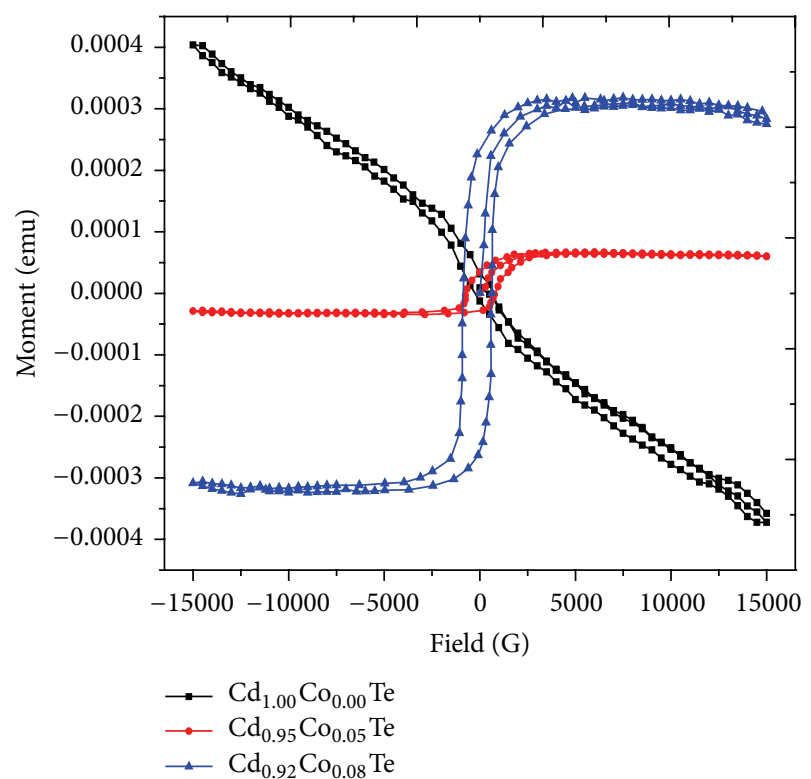

FIGURE 6: Plot of magnetization versus magnetic field of pure CdTe and Co doped CdTe alloy powders.

\section{Conclusions}

Co doped CdTe (with Co concentrations of 5 at.\% and 8 at.\%) powders were synthesized by single step solid-state reaction method. From X-ray diffraction, the crystal structure was confirmed to be cubic. The Co ions replaced the $\mathrm{Cd}$ ions without changing the CdTe crystal structure and the lattice parameter increased linearly with increase in Co level following Vegard's law. EDAX spectra revealed the existence of Co in $\mathrm{Cd}_{1-x} \mathrm{Co}_{x} \mathrm{Te}$ alloy powders. The reflectance measurement shows the increase in optical band gap energy with increase in Co level. The magnetization measurements clearly showed room temperature ferromagnetism in 5\% and $8 \%$ Co doped CdTe alloy powders.

\section{Acknowledgments}

The authors thank the Defense Research and Development Organization (DRDO), New Delhi, India, for providing financial support to carry out the present research work. The authors also express their thanks to SAIF-IIT Madras for providing the VSM and EDAX studies.

\section{References}

[1] J. K. Furdyna and J. Kossut, Semiconductors and Semimetals, vol. 25 of Diluted Magnetic Semiconductors, Academic Press, Boston, Mass, USA, 1988.

[2] S. J. Pearton, C. R. Abernathy, D. P. Norton et al., "Advances in wide bandgap materials for semiconductor spintronics," Materials Science and Engineering R, vol. 40, no. 4, pp. 137-168, 2003.

[3] H. Ohno, "Making nonmagnetic semiconductors ferromagnetic," Science, vol. 281, no. 5379, pp. 951-956, 1998. 
[4] S. A. Wolf, D. D. Awschalom, R. A. Buhrman et al., "Spintronics: a spin-based electronics vision for the future," Science, vol. 294, no. 5546, pp. 1488-1495, 2001.

[5] K. C. Hass and H. Ehrenreich, "Electronic and magnetic properties of II-VI diluted magnetic semiconductors," Journal of Crystal Growth, vol. 86, no. 1-4, pp. 8-14, 1990.

[6] D.-S. Chuu, Y.-C. Chang, and C.-Y. Hsieh, "Growth of CdMnS films by pulsed laser evaporation," Thin Solid Films, vol. 304, no. 1-2, pp. 28-35, 1997.

[7] H. Alawadhi, I. Miotkowski, A. Lewicki, A. K. Ramdas, S. Miotkowska, and M. McElfresh, "Magnetic susceptibility and compositional dependence of the energy gap in $\mathrm{Cd}_{1-x} \mathrm{Co}_{x} \mathrm{Te}$," Journal of Physics: Condensed Matter, vol. 14, no. 17, pp. 46114620, 2002.

[8] O. W. Shih, R. L. Aggarwal, T. Q. Vu, and P. Becla, "Bandedge magnetoreflectance and magnetization of $\mathrm{Cd}_{1-x} \mathrm{Co}_{x}$ Te," Solid State Communications, vol. 81, no. 3, pp. 245-249, 1992.

[9] V. G. Abramishvily, S. M. Ryabchenko, A. I. Savchuk, Y. G. Semenov, and A. V. Komarov, "Carrier-ion exchange interactions in crystals $\mathrm{Cd}_{1-x} \mathrm{Co}_{x} \mathrm{Te}$," Solid State Communications, vol. 101, no. 6, pp. 397-402, 1997.

[10] H. Alawadhi, I. Miotkowski, V. Souw, M. McElfresh, A. K. Ramdas, and S. Miotkowska, "Excitonic Zeeman effect in the zinc-blende II-VI diluted magnetic semiconductors $\mathrm{Cd}_{1-x} \mathrm{Y}_{x} \mathrm{Te}$ (Y=Mn, Co, and Fe)," Physical Review B, vol. 63, Article ID 155201, 10 pages, 2001.

[11] M. Zielinski, C. Rigaux, A. Lemaître, A. Mycielski, and J. Deportes, "Exchange interactions and magnetism of $\mathrm{Co}^{2+}$ in $\mathrm{Zn}_{1-x} \mathrm{Co}_{x}$ Te," Physical Review B, vol. 53, no. 2, pp. 674-684, 1996.

[12] M. Zielinski, C. Rigaux, A. Mycielski, and M. Menant, "Zeeman spectrum of the $1 s$ exciton in very diluted $\mathrm{Cd}_{1-x} \mathrm{Co}_{x} \mathrm{Te}$ compounds," Physical Review B, vol. 63, no. 3, Article ID 035202, 9 pages, 2000.

[13] R. D. Shannon, "Revised effective ionic radii and systematic studies of interatomic distances in halides and chalcogenides," Acta Crystallographica A, vol. 32, part 5, pp. 751-767, 1976.

[14] J. H. Kim, H. Kim, D. Kim, S. G. Yoon, and W. K. Choo, “Optical and magnetic properties of laser-deposited Co-doped $\mathrm{ZnO}$ thin films," Solid State Communications, vol. 131, no. 11, pp. 677-680, 2004.

[15] M. Bouloudenine, N. Viart, S. Colis, J. Kortus, and A. Dinia, "Antiferromagnetism in bulk $\mathrm{Zn}_{1-x} \mathrm{Co}_{x} \mathrm{O}$ magnetic semiconductors prepared by the coprecipitation technique," Applied Physics Letters, vol. 87, no. 5, Article ID 052501, 3 pages, 2005.

[16] Ü. Alver, E. Bacaksız, and E. Yanmaz, "Structural, magnetic and optical properties of Co-diffused CdTe thin films," Journal of Alloys and Compounds, vol. 456, no. 1-2, pp. 6-9, 2008. 

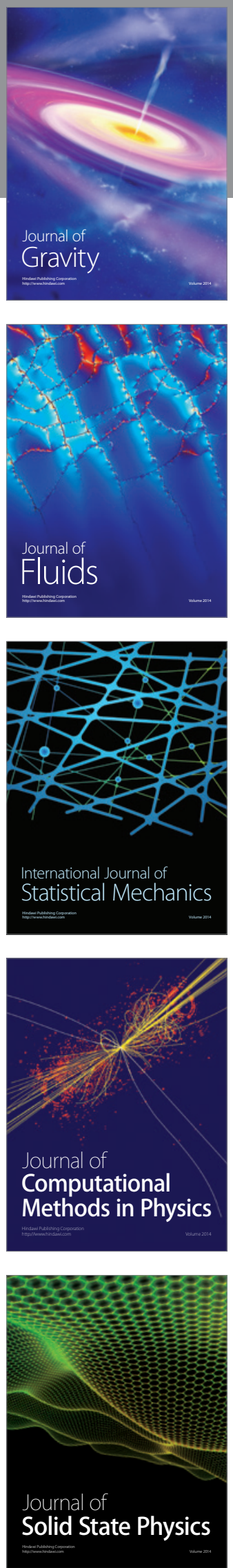

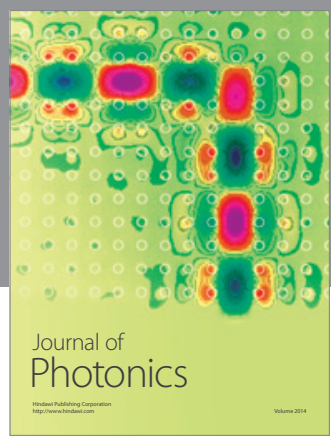

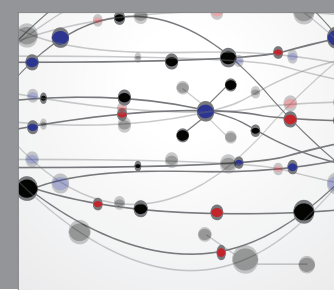

The Scientific World Journal

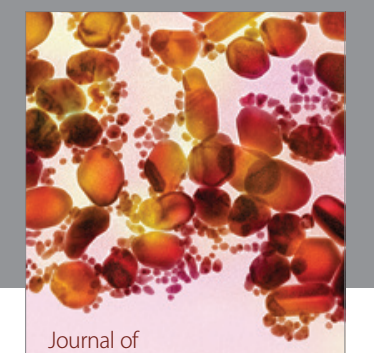

Soft Matter
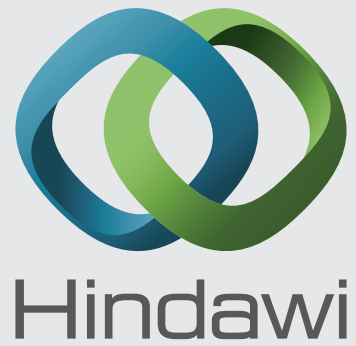

Submit your manuscripts at

http://www.hindawi.com
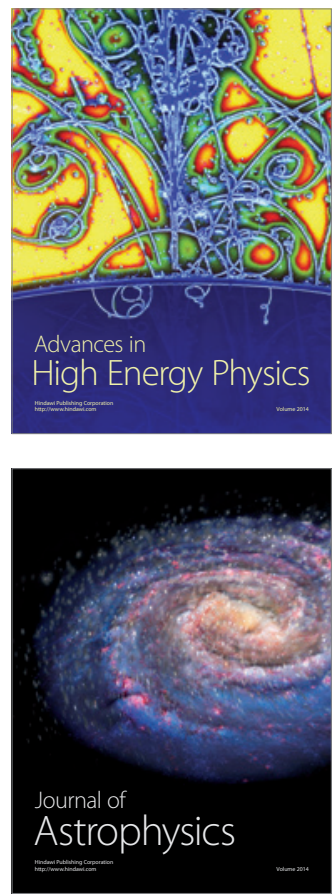
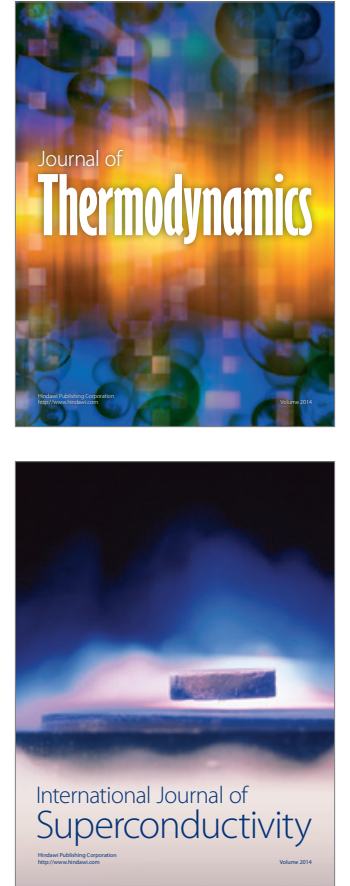
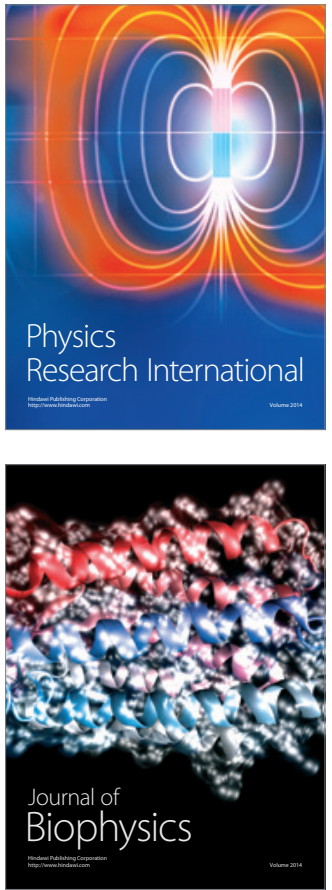
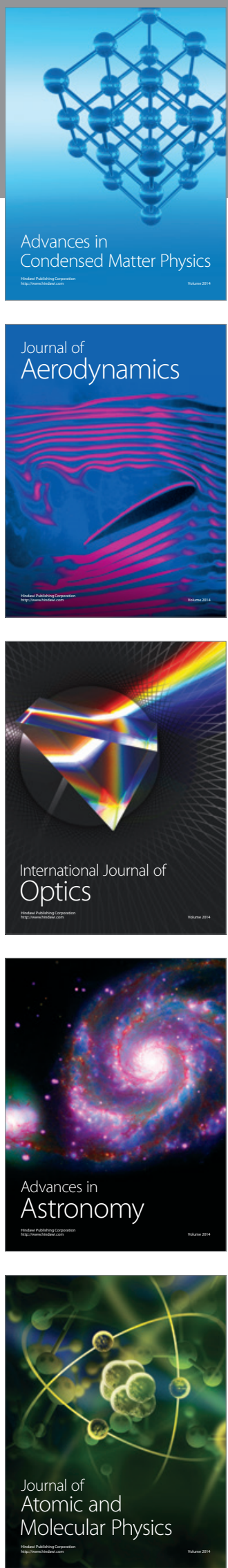